\title{
The Nitrogen Content Prediction Model of Cold Region Rice Canopy at the Tillering Stage Based on Hyperspectral Imaging
}

\author{
Yitong Liu, Yuzhu Song, Shuwen Wang, Yu Zhao* \\ School of Electric and Information, Northeast Agricultural University, Harbin, China \\ wswtr@163.com, 306434531@qq.com, 546473937@163.com, yuzhaoneau@outlook.com
}

\begin{abstract}
Keywords: Rice canopy, Hyper-spectrum, Nitrogen content, Tillering stage, Regression analysis
Abstract. In order to detect the nitrogen content of the rice canopy fast and nondestructively in the cold region, the hyperspectral technique was used to capture the rice canopy spectrum information at the tillering stage. After vegetation index was constructed by the characteristic waveband of high correlation, the linear regression prediction model and index combination prediction model was verified and analyzed. The result shows that the reflectance of rice canopy of tillering stage has significant differences in visible light band $(492 \mathrm{~nm} \sim 622 \mathrm{~nm})$ and near infrared band (greater than $770 \mathrm{~nm}$ ), and there is high correlation between rice canopy nitrogen content and 554nm, 736nm, $733 \mathrm{~nm}$ light bands. The accuracy and stability of the linear regression prediction model is better with SI (773), $\operatorname{DSI}_{(773,554)}$ as the variables, while the $R_{C}{ }^{2}$ is $0.771,0.724$ and the $R_{P}{ }^{2}$ is $0.789,0.773$ respectively. The two vegetation indices combination model constructed by $\operatorname{DSI}_{(773,554)}, \mathrm{NDSI}_{(773,554)}$ and the five vegetation indices combination model constructed by $\operatorname{DSI}_{(773,554)}, \operatorname{DSI}_{(773,736)}, \operatorname{RSI}_{(736,554)}$, $\operatorname{RSI}_{(773,554)}$, NDSI $(736,554)$ performed better than linear regression prediction model, while $R_{C}{ }^{2}$ is 0.773 , 0.795 and $R_{P}^{2}$ is $0.801,0.784$.
\end{abstract}

\section{Introduction}

The yield of rice in high latitude cold region, such as Heilongjiang province in China, is related to national food security. Nitrogen content is one of the essential elements for rice growth. Reasonable nitrogen fertilizer is important to increase the yield and quality of rice, and to avoid environmental problems caused by excessive fertilization[1]. Nowadays, there are more researches about nitrogen content level monitoring of corn and rice, but these studies analyze rice in cold region less.

Traditional nitrogen content detection methods have some disadvantages, such as high demand for experience, high cost, destructive sampling, and cannot meet the large farmland real-time monitoring because of time and space effect[2]. Hyperspectral remote sensing technique provides an effective way to detect crop nitrogen content level rapid and nondestructive. Tian analyzed the quantitative relationship between nitrogen concentration of rice canopy leaf and hyperspectral red edge[3]. Qin established the nitrogen content detection model based on the normalized difference vegetation index (NDVI) of rice canopy, using the early rice in Yingtan area (Jiangxi Province, China) as research object[4]. Liu summarized the response feature of rice canopy spectral reflectance and the first derivative reflectance under nitrogen stress[5]. Study of shao found that index model of first order differential reflectance at $738 \mathrm{~nm}$ could effectively predict the late rice canopy nitrogen content of the southern hilly region[6]. Wang proposed partial least squares mode(PLSR) based on normalized area band depth(BNA) and genetic algorithm(GA), according to the problem of data redundancy and model complexity when using hyperspectral PLSR to retrieve crop nitrogen content[7]. Li constructed rice canopy nitrogen monitoring model with high accuracy and generalization using random forest algorithm[8]. In this study, the rice in cold region of Heilongjiang province was taken as the object, and the purpose of the study is to establish the nitrogen content monitoring model to guide rice production and to apply nitrogen fertilizer rationally based on the hyperspectral remote sensing technique. 


\section{Experiment}

\section{Experiment design}

The experiment was carried out in Wuchang, Heilongjiang province in 2016, and Daohuaxiang was chosen as rice variety. There are 4 nitrogen level treatments $\left(\mathrm{N}_{0}, \mathrm{~N}_{1}, \mathrm{~N}_{2}, \mathrm{~N}_{3}\right)$, with 4 nitrogen fertilizer amount $\left(0,60,90,120 \mathrm{~kg} / \mathrm{hm}^{2}\right)$. Each level treatment is repeated 4 times, and the field distribution including 16 subfields is shown in Figure 1.

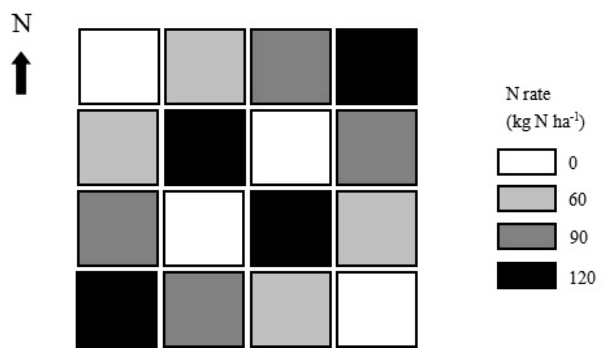

Fig. 1 Distribution of rice field

\section{Canopy image capturing}

The 710VP portable imaging spectrometer produced by SOC company was used, with wave length of $372-1038 \mathrm{~nm}$ and resolution of $4.68 \mathrm{~nm}$. The hyperspectral images of rice canopies at the tillering stage were captured at $10 \mathrm{am}$ to $14 \mathrm{pm}$ on $25^{\text {th }} \mathrm{June}, 2016$. The spectrometer probe was $100 \mathrm{~cm}$ away from top of the canopy vertically. Each subfield captured two images, and each image covered two complete plants of rice. After bright and dark current calibration, the hyperspectral images were captured and transferred.

\section{Acquisition of canopy leaf nitrogen content}

The 10 leaves of each rice plant at the top in each image were washed, dried, and crushed, then using the AA3 continuous flow analyzer to determine the amount of leaf nitrogen content.

\section{Extraction of canopy hyperspectral reflectance}

Five regions of interest (ROI) were selected from each rice plant in the hyperspectral image, and the average of five ROI reflectance was taken as the rice plant canopy reflectance. The canopy hyperspectral reflectance extraction is shown in Figure 2. There are 64 groups of data at the tillerring stage in this experiment. After removing the abnormal 2 groups of data, 42 groups of data are selected as the prediction set, and the remaining 20 groups of data are used as validation set.

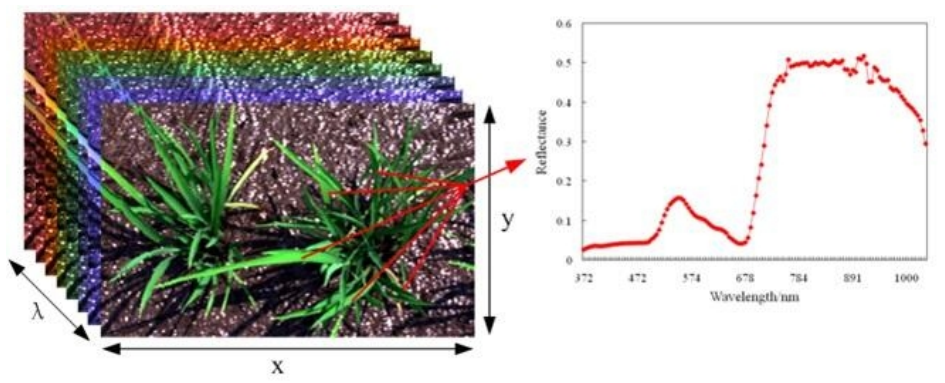

Fig.2 Extraction of hyperspectral reflectance

\section{Data analysis}

According to the relationship between canopy spectrum reflectance and nitrogen content, the single band index (SI), difference spectral index (DSI), ratio spectral index (RSI), normalized difference spectral index (NDSI), were selected to establish the prediction model. All the vegetation indices are computed as follows:

$$
S I=R_{\lambda_{1}}
$$




$$
\begin{aligned}
& D S I=R_{\lambda_{1}}-R_{\lambda_{2}} \\
& R S I=\frac{R_{\lambda_{1}}}{R_{\lambda_{2}}} \\
& N D S I=\frac{R_{\lambda_{1}}-R_{\lambda_{2}}}{R_{\lambda_{1}}+R_{\lambda_{2}}}
\end{aligned}
$$

\section{Result and Discussion}

\section{Analysis of rice canopy hyperspectral reflectance at the tillering stage}

As shown in Figure 3, rice canopy reflectance curves have similar trends at each nitrogen level. The reflectance curve rises slowly at violet waveband(372-455nm) and blue waveband(455-492nm). The reflectance curve increases rapidly at green waveband(492-577nm), and appears obvious peak at $554 \mathrm{~nm}$. The curve decreases fast at the yellow waveband $(577-597 \mathrm{~nm})$ and orange waveband(597-622nm), until appears obvious valley at red waveband(622-770nm) of $670 \mathrm{~nm}$. Then the curve increases fast at the near infrared waveband $(>770 \mathrm{~nm})$, and forms a high value platform. At the same time, the reflectance of green, yellow, orange waveband decreases in turn, while the reflectance of red and near infrared waveband increases, in the $\mathrm{N}_{0}-\mathrm{N}_{3}$ subfields. The reason is that chlorophyll content of rice leaves increases when nitrogen applications enhance. The higher chlorophyll content, the larger leaf area, and the stronger reflection at the near infrared waveband. So, different nitrogen content level can be distinguished according to canopy spectral reflectance in the visible light(492-622nm) and near infrared light(>770nm).

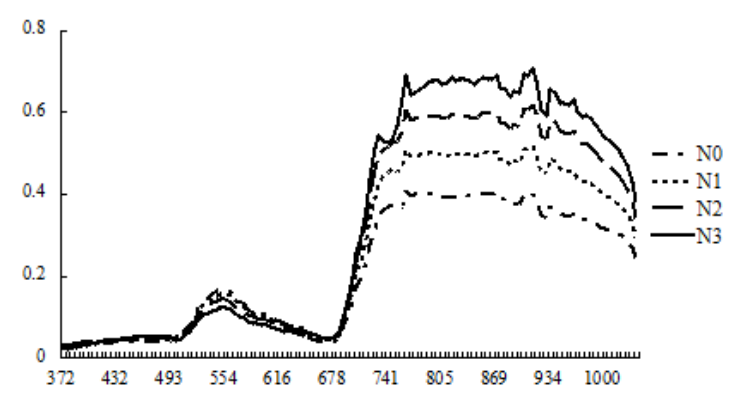

Fig.3 Hyperspectral reflectance of rice canopy of tillering stage at different nitrogen level

\section{Correlation analysis of canopy spectrum reflectance and nitrogen content}

The correlation analysis between the waveband reflectance and nitrogen content was made, and the correlation coefficient curve was calculated which is shown in Figure 4. The formula of computing coefficient is defined in (5), which $\mathrm{n}$ is sample size, $\mathrm{xi}$ and yi is sample value.

$$
r=\frac{\sum_{i=1}^{n}\left(x_{i}-\bar{x}\right)\left(y_{i}-\bar{y}\right)}{\sqrt{\sum_{i=1}^{n}\left(x_{i}-\bar{x}\right)^{2}\left(y_{i}-\bar{y}\right)^{2}}}
$$




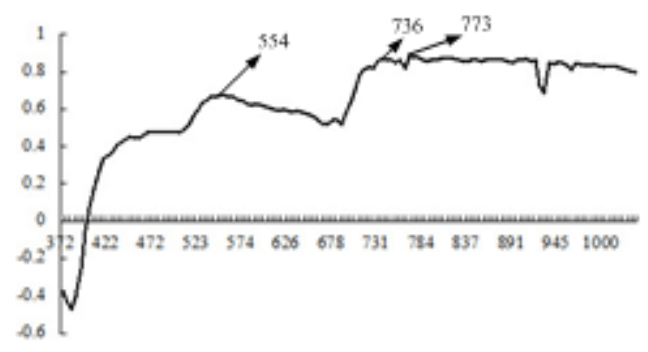

Fig.4 Correlation coefficient curve of hyperspectral reflectance and nitrogen content

As shown in Fig.4, the purple waveband is negatively correlated with nitrogen content, and the blue, near infrared waveband are positive associated with nitrogen content. The high correlation coefficients appear at 554nm (green light), 736nm (red light), 773nm (near infrared light). The coefficient value of near infrared light is greater than that of visible light.

\section{Prediction model of rice nitrogen content}

Prediction model of linear function, quadratic function, exponential function, logarithmic function, power function is established using regression analysis, which the vegetation index is independent variable $\mathrm{x}$, nitrogen content is dependent variable $\mathrm{y}$. The optimal prediction models of each vegetation index are shown Table 1 , in which $\mathrm{R}_{\mathrm{C}}{ }^{2}$ is prediction set coefficient and $\mathrm{R}_{\mathrm{P}}{ }^{2}$ is validation set coefficient. The precision and stability of model defined by SI and DSI are better than that of RSI and NDSI. The reason is that RSI and NDSI are not sensitive to nitrogen content when rice is at the tillering stage. The best prediction model is based on SI (773) and DSI (773, 554), the result is shown in Figure 5.

Table 1 Quantitative relationship between rice canopy nitrogen content and vegetation indices

\begin{tabular}{|c|c|c|c|}
\hline $\begin{array}{l}\text { Vegetation } \\
\text { Index }\end{array}$ & Prediction model & $R_{C}^{2}$ & $R_{P}^{2}$ \\
\hline $\mathrm{SI}_{(554)}$ & $\begin{array}{c}y=-23.677 x^{2}+14.544 x+ \\
1.189\end{array}$ & 0.499 & 0.397 \\
\hline $\mathrm{SI}_{(736)}$ & $y=3.891 x^{2}-0.368 x+2.110$ & 0.751 & 0.797 \\
\hline $\mathrm{SI}_{(773)}$ & $y=-1.597 x^{2}+3.929 x+1.164$ & 0.771 & 0.798 \\
\hline $\operatorname{DSI}_{(736,554)}$ & $y=8.978 x^{2}-1.663 x+2.386$ & 0.674 & 0.769 \\
\hline $\operatorname{DSI}_{(773,554)}$ & $y=1.336 x^{2}+1.638 x+1.945$ & 0.724 & 0.733 \\
\hline $\operatorname{DSI}_{(773,736)}$ & $\begin{array}{c}y=-68.536 x^{2}+16.733 x+ \\
2.017\end{array}$ & 0.636 & 0.403 \\
\hline $\operatorname{RSI}_{(736,554)}$ & $y=-0.18 x^{2}+1.316 x+0.433$ & 0.062 & 0.060 \\
\hline $\operatorname{RSI}_{(773,554)}$ & $\begin{array}{c}y=-0.107 x^{2}+1.003 x+ \\
0.565\end{array}$ & 0.175 & 0.126 \\
\hline $\operatorname{RSI}_{(773,736)}$ & $\begin{array}{c}y=-11.480 x^{2}+29.730 x- \\
16.184\end{array}$ & 0.421 & 0.227 \\
\hline $\operatorname{NDSI}_{(736,554)}$ & $\begin{array}{c}y=-10.934 x^{2}+12.622 x- \\
0.813\end{array}$ & 0.062 & 0.036 \\
\hline $\operatorname{NDSI}_{(773,554)}$ & $y=1.281 \ln x+3.507$ & 0.170 & 0.137 \\
\hline $\operatorname{NDSI}_{(773,736)}$ & $\begin{array}{c}y=-52.947 x^{2}+14.959 x+ \\
2.037\end{array}$ & 0.420 & 0.223 \\
\hline
\end{tabular}



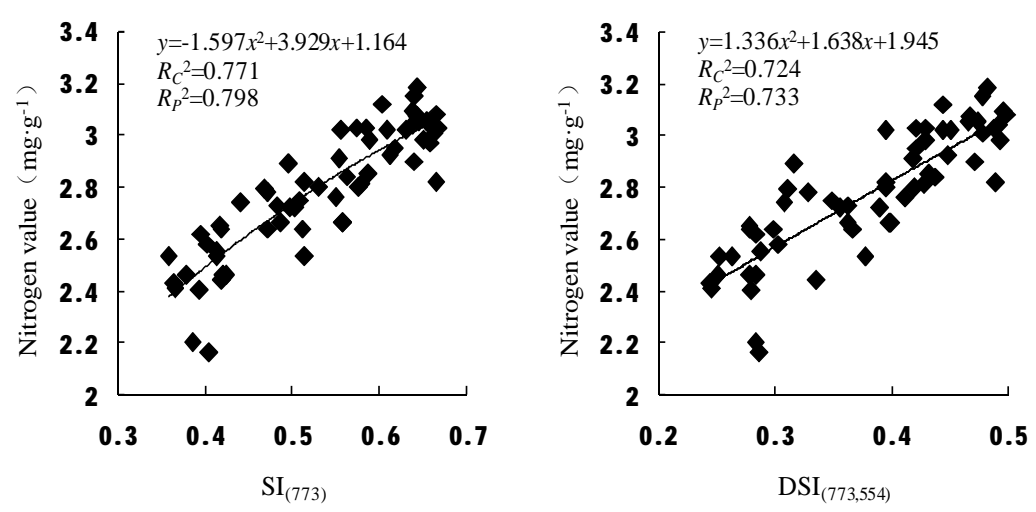

Fig. 5 The prediction model of linear regression

Multiple vegetation indices combination prediction models are established by 12 indices and nitrogen content using MAXR regression analysis. The combined prediction model is shown in Table 2. Since the number of vegetation index exceeds 9, the model cannot be optimized, and the list includes 2-9 vegetation indices combination prediction model. With the number of indices increases, $R_{\mathrm{c}}{ }^{2}$ increases, and $R_{\mathrm{c}}{ }^{2}$ remains unchanged until number of indices exceed 5 . With the number of indices increases, $R_{P}{ }^{2}$ decreases. Considering the model's precision and stability, two vegetation indices (DSI ${ }_{(773,554)}$, $\left.\operatorname{NDSI}_{(773,554)}\right)$ model and five vegetation indices $\left(\operatorname{DSI}_{(773,554)}, \operatorname{DSI}_{(773,736)}, \operatorname{RSI}_{(736,554)}, \operatorname{RSI}_{(773,554)}\right.$, $\left.\operatorname{NDSI}_{(736,554)}\right)$ model are optimal. The prediction result is shown in Figure 6.

Table 2. Quantitative relationship between rice canopy nitrogen content and vegetation index combination

\begin{tabular}{|c|c|c|c|}
\hline $\begin{array}{c}\text { Index } \\
\text { Amount }\end{array}$ & Prediction model & $R_{C}^{2}$ & $R_{P}^{2}$ \\
\hline 2 & $y=2.507+3.277 \operatorname{DSI}_{(773,554)}-1.734 \operatorname{NDSI}_{(773,554)}$ & 0.773 & 0.801 \\
\hline 3 & $y=2.532+2.427 \mathrm{SI}_{(773)}+0.399 \mathrm{RSI}_{(773,554)}-4.013 \mathrm{NDSI}_{(773,554)}$ & 0.780 & 0.783 \\
\hline 4 & $y=1.735+4.102 S \mathrm{I}_{(773)}-12.904 \mathrm{DSI}_{(773,736)}-1.94 \mathrm{RSI}_{(736,554)}+1.629 \mathrm{RSI}_{(773,554)}$ & 0.790 & 0.783 \\
\hline 5 & $y=3.295+5.8 \mathrm{DSI}_{(773,554)}-13.557 \mathrm{DSI}_{(773,736)}-1.485 \mathrm{RSI}_{(736,554)}+1.503 \mathrm{RSI}_{(773,554)}-4.939 \operatorname{NDSI}_{(736,554)}$ & 0.795 & 0.784 \\
\hline 6 & $y=31.372+2.941 \mathrm{SI}_{(736)}-4.7 \mathrm{RSI}_{(736,554)}+4.58 \mathrm{RSI}_{(773,554)}-29.127 \mathrm{RSI}_{(773,736)}-54.966 \mathrm{NDSI}_{(736,554)}+53.332 \operatorname{NDSI}_{(773,554)}$ & 0.798 & 0.772 \\
\hline 7 & $\begin{array}{c}y=34.165+4.255 \operatorname{DSI}_{(736,554)}-10.057 \operatorname{RSI}_{(736,554)}+9.64 \mathrm{RSI}_{(773,554)}-31.851 \mathrm{RSI}_{(773,736)}-155.838 \mathrm{NDSI}_{(736,554)}+ \\
155.866 \mathrm{NDSI}_{(773,554)}-100.446 \mathrm{NDSI}_{(773,736)}\end{array}$ & 0.798 & 0.750 \\
\hline 8 & $\begin{array}{c}y=27.486+4.907 \operatorname{DSI}_{(773,554)}-8.136 \operatorname{DSI}_{(773,736)}-10.7 \mathrm{RSI}_{(736,554)}+10.239 \mathrm{RSI}_{(773,554)}-25.251 \mathrm{RSI}_{(773,736)}- \\
167.7 \operatorname{NDSI}_{(736,554)}+167.85 \operatorname{NDSI}_{(773,554)}-124.7 \operatorname{NDSI}_{(773,736)}\end{array}$ & 0.799 & 0.752 \\
\hline 9 & $\begin{array}{c}y=27.611-4.063 \mathrm{SI}_{(554)}+4.625 \mathrm{SI}_{(773)}-7.689 \mathrm{DSI}_{(773,736)}-10.553 \mathrm{RSI}_{(736,554)}+10.1 \mathrm{RSI}_{(773,554)}-25.49 \mathrm{RSI}_{(773,736)}- \\
163.824 \mathrm{NDSI}_{(736,554)}+164.168 \mathrm{NDSI}_{(773,554)}-120.842 \mathrm{NDSI}_{(773,736)}\end{array}$ & 0.799 & 0.752 \\
\hline
\end{tabular}
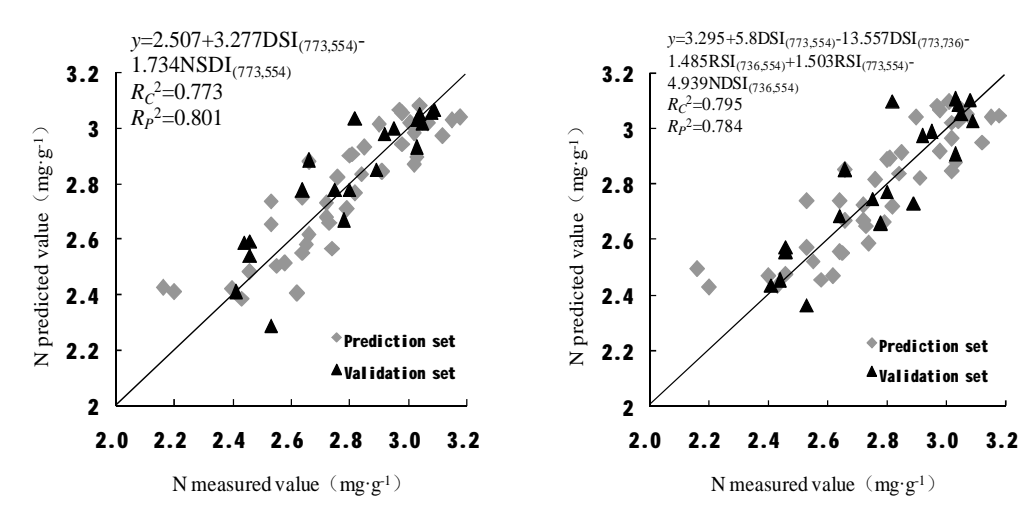

Fig. 6 The prediction model of vegetation index combination

\section{Conclusions}

Through the analysis of rice canopy reflectance curves, the vegetation indices are constructed by characteristic waveband. Linear regression prediction model is established by each vegetation index 
and vegetation index combination prediction model is constructed by MAXR regression method. After all the models are analyzed and validated, the conclusion is as follows:

1) Under different nitrogen application levels, there are significant differences of canopy reflectance in visible light (492-622nm) and near infrared light (>770nm).

2) The $554 \mathrm{~nm}, 736 \mathrm{~nm}$ and $773 \mathrm{~nm}$ waveband are highly correlated with rice canopy nitrogen content.

3) The precision and stability of linear regression model based on $\mathrm{SI}_{(773)}, \mathrm{DSI}_{(773,554)}$ is good, with $R_{C}{ }^{2}$ is $0.771,0.724$ and $R_{P}^{2}$ is $0.798,0.733$ respectively. The two index combination model based on $\operatorname{DSI}_{(773,554)}$, $\operatorname{DSI}_{(773,736)}$ is better than linear model, with $R_{C}{ }^{2}$ is 0.773 and $R_{P}{ }^{2}$ is 0.801 . The five index combination model based on $\operatorname{DSI}_{(773,554)}, \operatorname{DSI}_{(773,736)}, \operatorname{RSI}_{(736,554)}, \operatorname{RSI}_{(773,554)}, \operatorname{NDSI}_{(736,554)}$ is also better, with $R_{C}{ }^{2}$ is 0.795 and $R_{P}{ }^{2}$ is 0.784 .

\section{Acknowledgements}

The research is granted by National "863" Program (AA2013102303), Heilongjiang province Postdoctoral Research Foundation (LBH-Q13022), Heilongjiang Province Natural Science Foundation Project (C2015006), and Harbin science and technology innovation talent program (2015RQQXJ020).

\section{References}

[1]Lin Gong Wei, Shi Shuo et al. "Estimation of rice leaf nitrogen contents based on hyperspectral LIDAR". International Journal of Applied Earth Observation and Geoinformation, Vol. 44(2016), p. 136-143.

[2]Mizusaki, D., K. Umeki, and T. Honjo. "Development of models for estimating leaf chlorophyll and nitrogen contents in tree species with respect to seasonal changes." Photosynthetica ,Vol.51, No..4(2013),p.531-540.

[3]Tian, Yong Chao, et al. "Quantitative relationship between hyper-spectral red edge position and canopy leaf nitrogen concentration in rice. "Acta Agronomica Sinica. Vol.35,No.9(2009),p. 1681-1690.

[4]Qin, Xia, S. H. Wang, and L. H. Xue. "Nitrogen Nutrition Diagnosis of Early Rice with NDVI and Its Application for Nitrogen Topdressing Recommendation at Yingtan,Jiangxi Province." Scientia Agricultura Sinica. Vol.44,No.4,(2011),p.691-698.

[5]Liu, Tao Ju, et al. "An Analysis on Hyper-spectral Characteristics of Rice under Different Nitrogen Levels." Acta Agriculturae Universitatis Jiangxiensis. Vol.36,No.4 (2014),p.699-704

[6]Shao hua, et al. " Liu, Tao Ju, et al. "An Analysis on Hyper-spectral Characteristics of Rice under Different Nitrogen Levels." Acta Agriculturae Universitatis Jiangxiensis. Vol.37,No.6 (2015) p.975-981.

[7]Wang,yihan, Shi tiezhu, et al. "Partial Least Square Regression Model for Retrieving Paddy Rice Nitrogen Content with Band Depth Analysis and Genetic Algorithm". Remote Sensing Information. Vol.34(2015),p.973-979.

[8]Li Xuqing, Liu Xiangnan, Liu Meiling, et al. Random forest algorithm and regional applications of spectral inversion model for estimating canopy nitrogen concentration in rice[J]. Journal of Remote sensing, Vol.18,No.4(2014),p.923-933. 